\title{
Characterization of the xylose-transporting properties of yeast hexose transporters and their influence on xylose utilization
}

\author{
Tanja Hamacher, ${ }^{1}$ Jessica Becker, ${ }^{1}$ Márk Gárdonyi, ${ }^{2}$ \\ Bärbel Hahn-Hägerdal ${ }^{2}$ and Eckhard Boles ${ }^{1}$
}

\author{
Author for correspondence: Eckhard Boles. Tel: +49 21181 12778. Fax: +49 2118115370. \\ e-mail: boles@uni-duesseldorf.de
}

1 Institut für Mikrobiologie, Heinrich-Heine-Universität, Universitätsstr. 1, Geb. 26.12.01, D-40225 Düsseldorf, Germany

2 Department of Applied Microbiology, Lund University, 22100 Lund, Sweden

\begin{abstract}
For an economically feasible production of ethanol from plant biomass by microbial cells, the fermentation of xylose is important. As xylose uptake might be a limiting step for xylose fermentation by recombinant xyloseutilizing Saccharomyces cerevisiae cells a study of xylose uptake was performed. After deletion of all of the 18 hexose-transporter genes, the ability of the cells to take up and to grow on xylose was lost. Reintroduction of individual hexose-transporter genes in this strain revealed that at intermediate xylose concentrations the yeast high- and intermediate-affinity transporters Hxt4, Hxt5, Hxt7 and Gal2 are important xylose-transporting proteins. Several heterologous monosaccharide transporters from bacteria and plant cells did not confer sufficient uptake activity to restore growth on xylose. Overexpression of the xylose-transporting proteins in a xylose-utilizing PUA yeast strain did not result in faster growth on xylose under aerobic conditions nor did it enhance the xylose fermentation rate under anaerobic conditions. The results of this study suggest that xylose uptake does not determine the xylose flux under the conditions and in the yeast strains investigated.
\end{abstract}

Keywords: Saccharomyces cerevisiae, xylose uptake, glucose uptake, xylose fermentation, heterologous expression

\section{INTRODUCTION}

In common fermentation processes, the yeast Saccharomyces cerevisiae is widely used for biotechnological ethanol production. However, S. cerevisiae is only able to ferment hexose sugars, and not pentose sugars like xylose. Along with glucose, xylose is one of the major monosaccharide components of lignocellolosic biomass (Hayn et al., 1993). For an economically feasible industrial process for ethanol production from plant biomass in lignocellulose hydrolysates, it is necessary to ferment all sugars present (von Sivers \& Zacchi, 1995).

Efforts to establish a xylose-utilizing pathway in $S$. cerevisiae by insertion of the genes encoding xylose reductase and xylitol dehydrogenase from Pichia stipitis or other organisms have resulted in only poor ethanol production from xylose (Kötter \& Ciriacy, 1993; Tantirungkij et al., 1993; Walfridsson et al., 1995). Various steps, including the uptake of xylose, have been suggested to limit the metabolism of xylose in metabolically engineered S. cerevisiae (Kötter \& Ciriacy, 1993; Eliasson et al., 2000). Uptake of xylose by $S$. cerevisiae has been proposed to be mediated more or less unspecifically by its hexose-transport system. This is composed of a large family of 18 related transporter proteins called Hxts and additional sugar transporters with broader substrate specificity (Boles \& Hollenberg, 1997; Wieczorke et al., 1999). In xylose-uptake experiments with $S$. cerevisiae cells, two kinetically distinct uptake components with $K_{\mathrm{m}}$ values of about $0.19 \mathrm{M}$ and $1.5 \mathrm{M}$ were determined, demonstrating that the monosaccharide transport system in S. cerevisiae has nearly a 200-fold lower affinity for xylose than for glucose (Kotyk, 1967; Lagunas et al., 1982; Kötter \& Ciriacy, 1993).

To improve the xylose-uptake activity of $S$. cerevisiae it is necessary to identify the specific transporters mediating uptake of xylose. Additionally, heterologous xylose transporters from other organisms may be expressed in $S$. cerevisiae to improve xylose uptake. Using $S$. cerevisiae TMB3201, expressing a functional xylose utilization pathway but lacking the complete monosaccharidetransport system, we have characterized the xylosetransporting properties of monosaccharide transporters 
from yeast, bacteria and plants, by growth and $\left[{ }^{14} \mathrm{C}\right]$ xylose uptake. Especially the high-affinity glucose transporters of $S$. cerevisiae can mediate uptake of xylose. Nevertheless, overexpression of these transporters in the xylose-fermenting yeast strain PUA-X1,2 did not increase its growth rate on xylose nor its xylose fermentation rate.

\section{METHODS}

Yeast strains and growth conditions. Yeast strains were: CEN.PK2-1C (MATa leu2-3,112 ura3-52 trp1-289 his3-41 MAL2-8 ${ }^{\mathrm{C}}$ SUC2), EBY.VW4000 (MATa $\Delta$ hxt1-17 $\Delta$ gal2 $\Delta$ stl1 uagt1 $\Delta m p h 2$ amph3 leu2-3,112 ura3-52 trp1-289 his3-41 MAL2-8 SUC2) (Wieczorke et al., 1999), TMB3001 (MATa his3-41:: YIpXR/XDH/XK MAL2-8 SUC2) (Eliasson et al.,

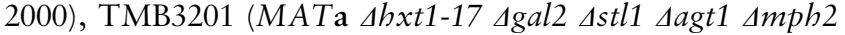
$\Delta m p h 3$ leu2-3,112 ura3-52 trp1-289 his3-41:: YIpXR/XDH/ XK MAL2-8 SUC2) (this work), PUA-X1,2 [his3-11 ura3-52 leu2::(LEU2:PDC1pr-XYL1, ADH1pr-XYL2)] (kindly provided by T. Weierstall, Düsseldorf). Synthetic media consisted of $6.7 \mathrm{~g} \mathrm{l}^{-1}$ Difco yeast nitrogen base (YNB) supplemented with amino acids and adenine, with or without uracil as a plasmid-selection marker, and with various carbon sources. Yeast cells were grown aerobically at $30^{\circ} \mathrm{C}$ on a rotary shaker or on agar plates. Xylose fermentation was investigated by incubating $50 \mathrm{ml}$ yeast cultures with a high cell density in sealed $50 \mathrm{ml}$ bottles under gentle stirring.

Construction of plasmids. The $0.4 \mathrm{~kb}$ SacI/SpeI MET25 promoter fragment of multicopy plasmid p426MET25 (Mumberg et al., 1994) was replaced by a $0.4 \mathrm{~kb}$ DNA fragment containing an HXT7 promoter fragment from -392 bp to -1 bp that was amplified by PCR with primers $\mathrm{P} 426 \mathrm{H} 7$ 1 (5'-CTAGAGCTCG TAGGAACAAT TTCGG-3') and P426H7-2 (5'-CGACTAGTGT GATGGTGATG GTGATGCATG TTAACTTTTT GATTAAAATT AAAAAAACTT$3^{\prime}$ ), and plasmid YEpkHXT7 (Krampe et al., 1998) as the template, resulting in plasmid p4H7. All monosaccharidetransporter genes were cloned by recombination-cloning into p4H7 using the strategies described by Wieczorke et al. (1999). The yeast hexose-transporter genes were amplified by wholecell PCR from strain FY1679 with GENEPAIRs primers obtained from Research Genetics. The Arabidopsis thaliana STP2 and STP3 ORFs were amplified from plasmids pUC19STP2 and pUC19STP3 using the primers F-ATSTP2 (5'-GGAATTCCAG CTGACCACCA TGGCTGTTGG TTCGATGA-3’), R-ATSTP2 (5'-GATCCCCGGG AATTGCCATG CTAGTCTTTG AAATATTTCT TCC-3'), F-ATSTP3 (5'-GGAATTCCAG CTGACCACCA TGGTAGCAGA AGAAGCAAG$\left.3^{\prime}\right)$ and R-ATSTP3 (5'-GATCCCCGGG AATTGCCATG TCAATGGCTA AGAATGGTG-3'), respectively. The $x y l E$ ORF was amplified from Escherichia coli strain JM109 with primers F-XYLE (5'-GGAATTCCAG CTGACCACCA TGAATACCCA GTATAATTCC-3') and R-XYLE (5'-GATCCCCGGG AATTGCCATG TTACAGCGTA GCAGTTTGTT G-3'). PCR products were further amplified with primers T3 (5'-GAATAAACAC AAAAACAAAA AGTTTTTTTA ATTTTAAGGA ATTCCAGCTG ACCACC-3') and T2ORFs (5'-GGGGGAGGGC GTGAATGTAA GCGTGACATA ACTAATTACA TGACTCGAGG ATCCCCGGGA ATTGCCATG-3'). The predicted A. thaliana xylose-proton symporter ORF was amplified from a seedling cDNA bank (Minet et al., 1992) with primers F7-AT5G (5'-TAATTTTAAT CAAAAAATGT GTTTTAAATC GAGGCCTGAC$\left.3^{\prime}\right)$ and R-AT5G (5'-CCCCGGGAAT TGCCATGTCA CTTCAAGATT TTTGATTC-3'), and the PCR product was further amplified with primers T71-ORFs (5'-AACACAAAAA CAAAAAGTTT TTTTAATTTT AATCAAAAA-3') and T2-ORFs. p4H7 was linearized with BamHI and HindIII and transformed into yeast strain EBY.VW4000, together with the PCR-amplified sugar-transporter genes, selecting for uracil prototrophy on a medium containing $2 \%$ maltose after homologous recombination in yeast. Plasmids were reisolated, amplified in E. coli SURE (Stratagene), and analysed by restriction enzyme mapping or sequencing (Seqlab). The resulting plasmids were named pTH[name of gene] (e.g. pTHHXT1, pTHHXT3). Construction of plasmids YEpkHXT7, p426MET25-HXT11 and pHL125-GAL2 is described elsewhere (Krampe et al., 1998; Wieczorke et al., 1999; Liang \& Gaber, 1996).

Other techniques. Molecular biology techniques were performed using published procedures (Sambrook et al., 1989). Xylose uptake was assayed as described previously (Weierstall et al., 1999). For the determination of the ethanol production rates, ethanol concentrations in yeast culture supernatants were measured using an enzymic test kit (Roche).

\section{RESULTS}

\section{Yeast hexose transporters mediating uptake of xylose}

The yeast strain TMB3001 (Eliasson et al., 2000), constructed by integrating a vector expressing $P$. stipitis XYL1 and XYL2 (encoding xylose reductase and xylitol dehydrogenase, respectively) and $S$. cerevisiae XKS1 (encoding xylulose kinase) into the genome of an $S$. cerevisiae CEN.PK strain, grew slowly in shaken-flask cultures with synthetic medium and $2 \%$ xylose as the carbon source (Fig. 1). In contrast, strain TMB3201, constructed by integrating the same plasmid into the CEN.PK-derived strain EBY.VW4000 (Wieczorke et al., 1999) lacking all its hexose-transporter genes, did not grow at all in the same medium. This demonstrates that one or more of the yeast hexose transporters is responsible for the uptake of xylose in $S$. cerevisiae.

In order to find the transporter(s) responsible, the $S$. cerevisiae hexose-transporter genes $H X T 1,3,4,5,8$, 9, $10,13,14$ and 15 were cloned by recombination-cloning into the multicopy expression vector $\mathrm{p} 4 \mathrm{H} 7$, driving expression from the very strong and constitutive $H X T 7^{1-392}$ bp promoter fragment. The resulting plasmids, the empty vector, and plasmids YEpkHXT7, p426MET25-HXT11 and pHL125-GAL2 (expressing the S. cerevisiae Hxt7, Hxt11 and Gal2 transporters, respectively), were transformed into the yeast strain TMB3201. The cells were plated onto agar plates of synthetic medium without uracil, containing $2 \%$ maltose as the carbon source. After replica-plating, all the transformants except those with the empty vector could grow on synthetic medium with glucose as the sole carbon source, indicating that all transporters were functionally expressed and able to mediate uptake of sugars into the cells.

To identify which hexose transporters are able to take up xylose, the growth rates of the transformants in liquid synthetic medium without uracil and with $2 \%$ xylose as the sole carbon source were determined. Only 

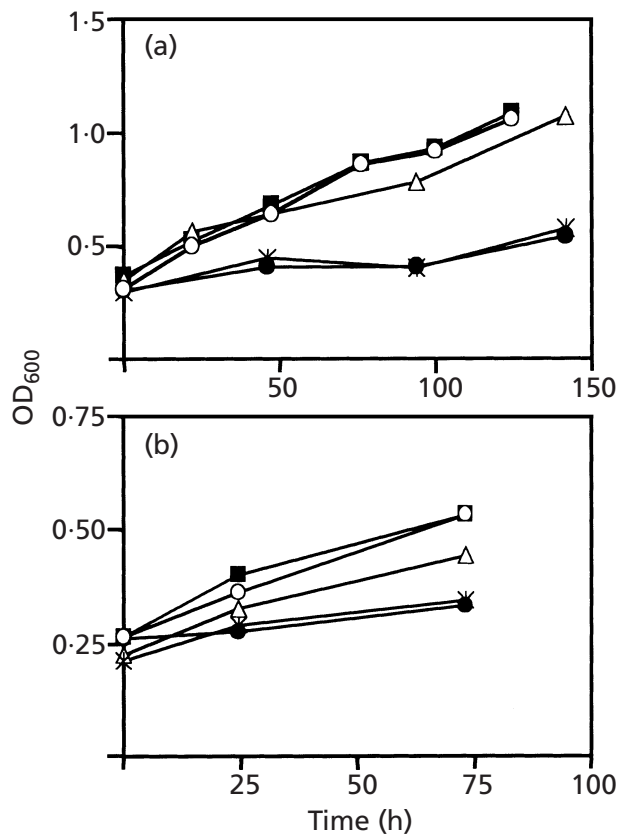

Fig. 1. Growth of $H X T$ mutant strains in xylose medium. Yeast transformants were pregrown in synthetic medium without uracil and with $2 \%$ maltose, washed twice and inoculated in synthetic medium without uracil and with $2 \%$ xylose at an initial $\mathrm{OD}_{600}$ of about 0.35 (a) or 0.25 (b). Cells were grown aerobically at $30{ }^{\circ} \mathrm{C}$ on a rotary shaker. Growth was monitored by measuring the $\mathrm{OD}_{600}$ of the cultures. Strains are: (a and b) TMB3001(p4H7) (a), TMB3201(p4H7) (0); (a) TMB3201(pTHHXT1) (X), TMB3201(YEpkHXT7) ( $\triangle$ ), TMB3201(pHL125-GAL2) (O); (b) TMB3201(pTHHXT4) (O),

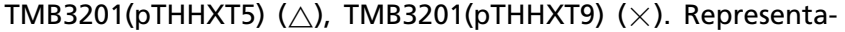
tive results of at least two independent experiments for each strain are shown.

transformants expressing the Hxt4, Hxt5, Hxt7 or Gal2 transporters were able to grow in the xylose medium (Fig. 1). In contrast, transformants expressing the transporters Hxt1, 3, 8, 9, 10, 11, 13, 14 and 15 did not grow with $2 \%$ xylose. Our results indicated that Hxt4, 5 and 7, and Gal2, are able to mediate the uptake of xylose into $S$. cerevisiae cells. We did not analyse Hxt2 as it was not possible to clone it behind the HXT7 promoter fragment on vector $\mathrm{p} 4 \mathrm{H} 7$. Hxt6, Hxt16 and Hxt17 were not included because they are almost identical to Hxt7, Hxt15 and Hxt13, respectively. HXT12 is a pseudogene (Wieczorke et al., 1999).

\section{Determination of xylose-uptake activities}

To confirm the results obtained in the growth tests, short-term (60 s) xylose uptake assays were performed with radiolabelled xylose. Xylose uptake was linear for at least $60 \mathrm{~s}$ under the conditions used (data not shown). Transformants of strain TMB3201 expressing Hxt4, Hxt5, Hxt7 or Gal2, or containing the empty vector $\mathrm{p} 4 \mathrm{H} 7$, were grown into the exponential growth phase in synthetic medium without uracil and with $2 \%$ maltose. As expected, the hexose-transport-deficient strain TMB3201 with the empty vector was not able to take up

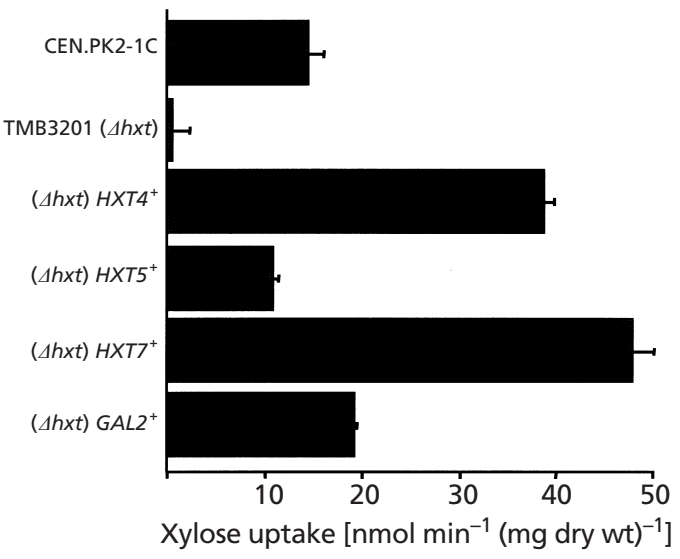

Fig. 2. Xylose uptake in $H X T$ mutant strains. Yeast transformants CEN.PK2-1C(p4H7), TMB3201(p4H7), TMB3201 (pTHHXT4), TMB3201(pTHHXT5), TMB3201(YEpkHXT7) and TMB3201(pHL125-GAL2) were grown into the exponential growth phase in synthetic medium without uracil and with $2 \%$ maltose before harvesting. Xylose uptake was measured for $1 \mathrm{~min}$ with $50 \mathrm{mM} \mathrm{D}-\left[\mathrm{U}-{ }^{14} \mathrm{C}\right] \mathrm{xylose}$. The results are means of three determinations \pm SEM.

xylose (Fig. 2). In contrast, expression of $\mathrm{Hxt} 4,5$ and 7 , and Gal2, conferred xylose uptake activities upon the strain. Xylose uptake activity with $50 \mathrm{mM}$ radiolabelled xylose was highest for the strain expressing Hxt7 [48 nmol $\min ^{-1}$ ( $\mathrm{mg}$ dry weight $)^{-1}$ ] and lowest for the strain expressing Hxt5 (11 nmol min ${ }^{-1}$ (mg dry weight $)^{-1}$ ] (Fig. 2). The parental strain CEN.PK2-1C transformed with the empty vector $\mathrm{p} 4 \mathrm{H} 7$ exhibited an uptake activity of 15 nmol $\min ^{-1}$ (mg dry weight $)^{-1}$.

\section{Expression of heterologous monosaccharide transporters in S. cerevisiae}

The $S$. cerevisiae hexose transporters solely mediate facilitated diffusion of their substrates down a concentration gradient (Lagunas, 1993). In contrast, several other yeasts, plant cells and bacterial cells can actively transport hexoses and pentoses by proton-symport mechanisms against concentration gradients (Boles \& Hollenberg, 1997; Büttner \& Sauer, 2000). Moreover, these proton-symport systems normally exhibit much higher affinities for their substrates. We therefore investigated whether such heterologous transporters could restore growth on xylose to the monosaccharidetransport-deficient yeast strain TMB3201.

The E. coli $x y l E$ gene encodes a xylose-proton symporter (Davis \& Henderson, 1987). The hexose-proton symporters Hup1 from Chlorella kessleri, and Stp2 and Stp3 from A. thaliana, have been shown to mediate uptake not only of various hexoses but also of xylose (reviewed by Büttner \& Sauer, 2000). Moreover, after completion of the A. thaliana genome sequencing project, a sequence has been found on chromosome 5 encoding a predicted xylose-proton-symporter-like protein targeted to chloroplasts (accession number At5g59250). The $x y l E, S T P 2$ and STP3 ORFs, as well as 
the putative xylose-proton symporter sequence lacking the 31 amino acids encoding the chloroplast-targeting sequence, were cloned into the multicopy expression vector $\mathrm{p} 4 \mathrm{H} 7$. After transformation of the resulting plasmids and plasmid pNEV-E expressing HUP1 behind the yeast PMA1 promoter (Robl et al., 2000) into strain TMB3201, Stp2, Stp3 and Hup1, but not - as expected - XylE and the putative A. thaliana xylose-proton symporter, supported growth of the yeast transformants on synthetic medium with $2 \%$ glucose (data not shown). However, none of the transporters restored growth in synthetic medium with $2 \%$ xylose, indicating that they are not able to take up xylose into the yeast cells. Moreover, we transformed strain TMB3201 with the $P$. stipitis genomic DNA library YEpTW (Weierstall et al., 1999) and an A. thaliana seedling cDNA bank (Minet et al., 1992), and selected the transformants for growth in synthetic medium with $2 \%$ xylose. From a total of more than 18000 and 10000 transformants, respectively, we could not find any transformant able to utilize and to grow on xylose.

\section{Xylose fermentations and growth tests with HXT- overexpressing strains}

The $S$. cerevisiae PUA strains were selected for faster growth on xylulose media (Porep, 1987; Kötter \& Ciriacy, 1993). Subsequently, the P. stipitis XYL1 and $X Y L 2$ ORFs under control of the S. cerevisiae PDC1 and $A D H 1$ promoters, respectively, were chromosomally integrated in these strains, resulting in PUA-X1,2. In order to see whether overexpression of xylose-transporting proteins could increase the ethanol production rate from xylose by recombinant $S$. cerevisiae strains, pTHHXT5, YEpkHXT7, pHL125-GAL2 and the empty vector were transformed into the PUA-X1,2 strain. The transformants were pre-grown on synthetic medium with $2 \%$ xylose to an $\mathrm{OD}_{600}$ of $1 \cdot 9$. Cells were harvested by centrifugation and washed twice with $2 \%$ xylose medium. They were then resuspended in 0.1 vol. of the volume of the same medium, incubated at $30^{\circ} \mathrm{C}$ and the ethanol concentration in the medium determined at different time points. As can be seen in Fig. 3, overexpression of the monosaccharide transporters did not increase the ethanol production rate. Instead, it seemed that ethanol production was even slightly delayed, especially in the case of the Gal2-overproducing transformants.

Under anaerobic conditions, the recombinant PUA strains cannot grow on xylose medium (Kötter \& Ciriacy, 1993; data not shown). Therefore, the growth rates of the transformants were determined under aerobic conditions in shaken-flask cultures with synthetic medium and $2 \%$ xylose. Growth on xylose of the recombinant PUA strains was twice as fast as that of the CEN.PK-derived strains (doubling times about 13 and $29 \mathrm{~h}$, respectively). However, the growth rates of the transformants overexpressing the xylose-transporting proteins Hxt4, Hxt7 and Gal2 were the same as those of the transformants with the empty vector. Moreover, no ethanol could be detected in the culture supernatants of

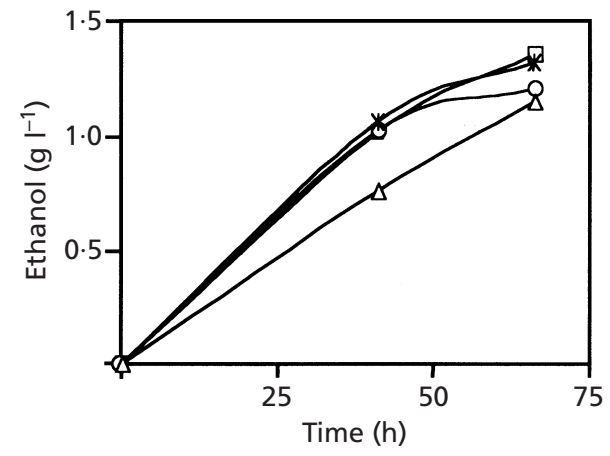

Fig. 3. Ethanol production of PUA-X1,2 cells overexpressing xylose transporters. Transformants TMB3001(p4H7) (X), TMB3201(pTHHXT5) ( $\square$ ), TMB3201(YEpkHXT7) (O) and TMB3201(pHL125-GAL2) ( $\triangle$ ) were pregrown in synthetic medium without uracil and with $2 \%$ xylose to an $\mathrm{OD}_{600}$ of 1.9 , washed twice, resuspended in $0.1 \mathrm{vol}$. of the same medium, and incubated under (semi-)anaerobic conditions at $30^{\circ} \mathrm{C}$. Ethanol concentrations in the culture supernatants were determined at various time points. Representative results of at least two independent experiments for each strain are shown.

any of the transformants. Our results indicate that xylose uptake does not determine xylose flux, at least not as the only step in xylose utilization.

\section{DISCUSSION}

We have shown that after deletion of all of the 18 hexose-transporter genes from a recombinant yeast strain able to utilize the pentose sugar xylose, its ability to take up and to grow on xylose is lost. This is, for the first time, a genetical proof that the yeast hexose-uptake system also mediates uptake of xylose. It confirms earlier results obtained by kinetic and physiological analysis of xylose uptake in yeast cells (Serrano \& de la Fuente, 1974; Busturia \& Lagunas, 1986; Kötter \& Ciriacy, 1993; Meinander \& Hahn-Hägerdal, 1997). After overexpression of individual hexose-transporter genes in the xylose uptake-deficient yeast strain, we could demonstrate that at xylose concentrations of $2 \%$ only Hxt4, Hxt5, Hxt7 and Gal2 are able to transport the sugar in significant amounts. Hxt7 and Gal2 are high-affinity hexose transporters whereas $\mathrm{Hxt} 4$ and Hxt5 are moderately low in affinity for glucose (Reifenberger et al., 1997; Diderich et al., 2001). We have not tested Hxt2 and Hxt6. However, as Hxt6 is nearly identical to Hxt7, and Hxt2 shows transport kinetics similar to $\mathrm{Hxt} 4$, we propose that also these transporters can mediate uptake of xylose. However, the low-affinity hexose transporters Hxt1 and Hxt3, and also the normally very weakly expressed transporters Hxt8 to Hxt17 were not able to transport xylose, at least in amounts sufficient to support growth.

The results were obtained after constitutive overexpression of the hexose-transporter genes. Normally, these genes are regulated by the presence and concentration of glucose or galactose (reviewed by Boles \& Hollenberg, 1997; Özcan \& Johnston, 1999). Whereas expression of 
HXT1 to HXT4 requires the presence of glucose in the medium, GAL2 is only expressed in the presence of galactose and absence of glucose. In contrast, HXT5 and HXT7 are also expressed in the absence of glucose (Schulte et al., 2000; Diderich et al., 1999, 2001). This means that in recombinant xylose-utilizing $S$. cerevisiae cells during growth on xylose mainly Hxt5 and Hxt7 may contribute to the uptake of the sugar.

None of the heterologous sugar transporters was able to support growth on xylose. In the case of E. coli XylE and the predicted $A$. thaliana xylose-proton symporter, we cannot exclude that these transporters are not correctly targeted to the plasma membrane in a functional form, as has been observed for several heterologous membrane proteins in yeast (Villalba et al., 1992; Jahn et al., 2002; our own unpublished results). However, C. kessleri Hup1 and A. thaliana Stp2 and Stp3 were functionally expressed in the plasma membrane, as they supported growth of the hexose-transport-deficient yeast strain on glucose. Growth of these strains on glucose was significantly lower than parental growth, indicating that the capacity of the heterologous transporters was very low. For all three transporters, xyloseuptake activity has been demonstrated (Büttner \& Sauer, 2000). Therefore, we propose that the xylose-uptake activity mediated by Hup1, Stp2 and Stp3 is too low to support growth on xylose.

Overexpression of the xylose-transporting hexose transporters in a xylose-utilizing $S$. cerevisiae PUA strain did not result in faster growth on $2 \%$ xylose medium under aerobic conditions, nor did it result in an increase of the ethanol production rate under (semi-)anaerobic conditions. This is in contrast to the recent observation that under anaerobic conditions in chemostat cultures with mineral medium increasing the xylose concentration in the feed enhanced the xylose flux, suggesting that transport limits flux (Eliasson et al., 2000). However, in that study a different, CEN.PK-derived yeast strain was used. Indeed, the PUA strain that we have used has been selected for faster growth on xylulose medium (Porep, 1987; Kötter \& Ciriacy, 1993). One of its new properties is a higher endogenous level of xylulose kinase activity. The growth rate on xylose was more than twofold higher for the PUA-derived recombinant strain than for the CEN.PK-derived strain. Interestingly, the xyloseuptake activity was also about twofold higher in PUA cells (data not shown). This could mean that in the PUA strain during selection for faster growth on xylulose the sugar-uptake system was one of the steps that were optimized. Unfortunately, the parental strain is no longer available to confirm this assumption. Nevertheless, it seems that in different yeast strains different factors might contribute to limited xylose fermentation, with xylose uptake being one of them.

\section{ACKNOWLEDGEMENTS}

We thank Professor C. P. Hollenberg for his kind support. We also thank T. Weierstall, I. Robl, W. Tanner, R. Gaber, M. Büttner and N. Sauer for their kind gifts of yeast strains and plasmids. This work was supported by a grant from the European Commission (QLK3-CT-1999-00080) to E.B. and B.H.-H.

\section{REFERENCES}

Boles, E. \& Hollenberg, C. P. (1997). The molecular genetics of hexose transport in yeasts. FEMS Microbiol Rev 21, 85-111.

Busturia, A. \& Lagunas, R. (1986). Catabolite inactivation of the glucose transport system in Saccharomyces cerevisiae. J Gen Microbiol 132, 152-160.

Büttner, M. \& Sauer, N. (2000). Monosaccharide transporters in plants: structure, function and physiology. Biochim Biophys Acta $1465,263-274$.

Davis, E. O. \& Henderson, P. J. (1987). The cloning and DNA sequence of the gene $x y l E$ for xylose-proton symport in Escherichia coli K12. J Biol Chem 262, 13928-13932.

Diderich, J. A., Schepper, M., van Hoek, P. \& 8 other authors (1999). Glucose uptake kinetics and transcription of HXT genes in chemostat cultures of Saccharomyces cerevisiae. J Biol Chem 274, 15350-15359.

Diderich, J. A., Merijn Schuurmans, J., Van Gaalen, M. C., Kruckeberg, A. L. \& Van Dam, K. (2001). Functional analysis of the hexose transporter homologue HXT5 in Saccharomyces cerevisiae. Yeast 18, 1515-1524.

Eliasson, A., Christensson, C., Wahlbom, C. F. \& Hahn-Hägerdal, B. (2000). Anaerobic xylose fermentation by recombinant $\mathrm{Sac}$ charomyces cerevisiae carrying XYL1, XYL2, and XKS1 in mineral medium chemostat cultures. Appl Environ Microbiol 66, 3381-3386.

Hayn, M., Steiner, W., Klinger, R., Steinmüller, H., Sinner, M. \& Esterbauer, H. (1993). Basic research and pilot plant studies on the enzymatic conversion of lignocellulosics. In Bioconversion of Forest and Agricultural Plant Residues, pp. 33-72. Edited by J. N. Saddler. Wallingford, UK: CAB International.

Jahn, T. P., Schulz, A., Taipalensuu, J. \& Palmgren, M. G. (2002). Post-translational modification of plant plasma membrane $\mathrm{H}^{+}$ATPase as a requirement for functional complementation of a yeast transport mutant. J Biol Chem 277, 6353-6358.

Kötter, P. \& Ciriacy, M. (1993). Xylose fermentation by Saccharomyces cerevisiae. Appl Microbiol Biotechnol 38, 776-783.

Kotyk, A. (1967). Mobility of the free and the loaded monosaccharide carrier in Saccharomyces cerevisiae. Biochim Biophys Acta 135, 112-119.

Krampe, S., Stamm, O., Hollenberg, C. P. \& Boles, E. (1998). Catabolite inactivation of the high-affinity hexose transporters Hxt6 and Hxt7 of Saccharomyces cerevisiae occurs in the vacuole after internalization by endocytosis. FEBS Lett 441, 343-347.

Lagunas, R. (1993). Sugar transport in Saccharomyces cerevisiae. FEMS Microbiol Rev 104, 229-242.

Lagunas, R., Dominguez, C., Busturia, A. \& Saez, M. J. (1982). Mechanisms of the appearance of the Pasteur effect in Saccharomyces cerevisiae: inactivation of sugar transport systems. J Bacteriol 152, 19-25.

Liang, H. \& Gaber, R. F. (1996). A novel signal transduction pathway in Saccharomyces cerevisiae defined by Snf3-regulated expression of HXT6. Mol Biol Cell 7, 1953-1966.

Meinander, N. Q. \& Hahn-Hägerdal, B. (1997). Influence of cosubstrate concentration on xylose conversion by recombinant, XYL1-expressing Saccharomyces cerevisiae: a comparison of different sugars and ethanol as cosubstrates. Appl Environ Microbiol 63, 1959-1964. 
Minet, M., Dufour, M.-E. \& Lacroute, F. (1992). Complementation of Saccharomyces cerevisiae auxotrophic mutants by Arabidopsis thaliana cDNAs. Plant J 2, 417-422.

Mumberg, D., Müller, R. \& Funk, M. (1994). Regulatable promotors of Saccharomyces cerevisiae: comparison of transcriptional activity and their use for heterologous expression. Nucleic Acids Res 22, 5767-5768.

Özcan, S. \& Johnston, M. (1999). Function and regulation of yeast hexose transporters. Microbiol Mol Biol Rev 63, 554-569.

Porep, H. J. (1987). Xyluloseverwertung bei Saccharomyces cerevisiae. PhD thesis, University of Düsseldorf.

Reifenberger, E., Boles, E. \& Ciriacy, M. (1997). Kinetic characterization of individual hexose transporters of Saccharomyces cerevisiae and their relation to the triggering mechanisms of glucose repression. Eur J Biochem 245, 324-333.

Robl, I., Grassl, R., Tanner, W. \& Opekarova, M. (2000). Properties of a reconstituted eukaryotic hexose/proton symporter solubilized by structurally related non-ionic detergents: specific requirement of phosphatidylcholine for permease stability. Biochim Biophys Acta 1463, 407-418.

Sambrook, J., Fritsch, E. F. \& Maniatis, T. (1989). Molecular Cloning: a Laboratory Manual, 2nd edn. Cold Spring Harbor, NY: Cold Spring Harbor Laboratory.

Schulte, F., Wieczorke, R., Hollenberg, C. P. \& Boles, E. (2000). The HTR1 gene is a dominant negative mutant allele of MTH1 and blocks Snf3- and Rgt2-dependent glucose signaling in yeast. J Bacteriol 182, 540-542.

Serrano, R. \& de la Fuente, G. (1974). Regulatory properties of the constitutive hexose transport in Saccharomyces cerevisiae. Mol Cell Biochem 5, 161-171.

Tantirungkij, M., Nakashima, N., Seki, T. \& Yoshida, T. (1993). Construction of xylose-assimilating Saccharomyces cerevisiae. J Ferm Bioeng 75, 83-88.

Villalba, J. M., Palmgren, M. G., Berberian, G. E., Ferguson, C. \& Serrano, R. (1992). Functional expression of plant plasma membrane $\mathrm{H}^{+}$-ATPase in yeast endoplasmic reticulum. $\mathrm{J}$ Biol Chem 267, 12341-12349.

von Sivers, M. \& Zacchi, G. (1995). A techno-economical comparison of three processes for the production of ethanol from pine. Bioresour Technol 51, 43-52.

Walfridsson, M., Hallborn, J., Penttilä, M., Keränen, S. \& HahnHägerdal, B. (1995). Xylose-metabolizing Saccharomyces cerevisiae strains overexpressing TKL1 and TAL1 genes encoding the pentose phosphate pathway enzymes transketolase and transaldolase. Appl Environ Microbiol 61, 4184-4190.

Weierstall, T., Hollenberg, C. P. \& Boles, E. (1999). Cloning and characterization of three genes (SUT1-3) encoding glucose transporters of the yeast Pichia stipitis. Mol Microbiol 31, 871-883.

Wieczorke, R., Krampe, S., Weierstall, T., Freidel, K., Hollenberg, C. P. \& Boles, E (1999). Concurrent knock-out of at least 20 transporter genes is required to block uptake of hexoses in Saccharomyces cerevisiae. FEBS Lett 464, 123-128.

Received 29 April 2002; revised 7 June 2002; accepted 13 June 2002. 